\title{
ORBITS OF FAMILIES OF VECTOR FIELDS AND INTEGRABILITY OF DISTRIBUTIONS
}

\author{
HÉCTOR J. SUSSMANN
}

\begin{abstract}
Let $D$ be an arbitrary set of $C^{\infty}$ vector fields on the $C^{\infty}$ manifold $M$. It is shown that the orbits of $D$ are $C^{\infty}$ submanifolds of $M$, and that, moreover, they are the maximal integral submanifolds of a certain $C^{\infty}$ distribution $P_{D^{*}}$. (In general, the dimension of $P_{D^{(m)}}\left(m\right.$ will not be the same for all $m \in M_{0}$ ) The second main result gives necessary and sufficient conditions for a distribution to be integrable. These two results imply as easy corollaries the theorem of Chow about the points attainable by broken integral curves of a family of vector fields, and all the known re sults about integrability of distributions (i.e. the classical theorem of Frobenius for the case of constant dimension and the more recent work of Hermann, Nagano, Lobry and Matsuda). Hermann and Lobry studied orbits in connection with their work on the accessibility problem in control theory. Their method was to apply Chow's theorem to the maximal integral submanifolds of the smallest distribution $\Delta$ such that every vector field $X$ in the Lie algebra generated by $D$ belongs to $\Delta$ (i.e. $X(m) \in \Delta(m)$ for every $m \in M$ ). Their work therefore requires the additional assumption that $\Delta$ be integrable. Here the opposite approach is taken. The orbits are studied directly, and the integrability of $\Delta$ is not assumed in proving the first main result. It turns out that $\Delta$ is integrable if and only if $\Delta=P_{D}$, and this fact makes it possible to derive a characterization of integrability and Chow's theorem. Therefore, the approach presented here generalizes and unifies the work of the authors quoted above.
\end{abstract}

1. Introduction. Let $D$ be a set of $C^{\infty}$ vector fields on the $C^{\infty}$ manifold $M$. We are interested in studying the $D$-orbits. Precisely, let $G$ be the "group" of local diffeomorphisms generated by the one-parameter groups whose infinitesimal generators are the elements of $D$. The D-orbits are the orbits of the action of $G$. Our main result (Theorem 4.1 ) states that the $D$-orbits (with a natural topology that that we define in $\$ 2$ ) are $C^{\infty}$ submanifolds of $M$. Moreover, we show how to associate with $D$ a $C^{\infty}$ distribution $P_{D}$ (i.e. a mapping which to each $m \in M$ assigns a linear subspace of the tangent space to $M$ at $m$; the dimension of this subspace may vary with $m$, and the mapping is supposed to be $C^{\infty}$ in a sense made precise in \$3). This distribution has the property that through every point of $M$ there passes a maximal integral manifold of $P_{D}$ and that, moreover, these maximal integral manifolds are precisely the orbits of $D$.

Received by the editors June 13, 1972.

AMS (MOS) subject classifications (1970). Primary 34C35, 34C40, 58A30, 49E15, 34H05, 93B05. 
The orbits of an arbitrary set $D$ of vector fields have been studied in the literature because of their importance in Control Theory (cf. Hermann [4], Lobry [5], Sussmann and Jurdjevic [8]). The method that has been used so far relies on a theorem by Chow [2], and on the theory of integral submanifolds of distributions. Let $D^{*}$ denote the smallest set of vector fields which contains $D$ and is closed under Lie brackets. Chow's theorem says that if $D^{*}(m)$ has maximal rank for each $m \in M$, then the orbits of $D$ are precisely the connected components of $M$ (here we use the notation $\left.D^{*}(m)=\left\{X(m): X \in D^{*}\right\}\right)$. Even if the assumption of Chow's theorem is not satisfied, it is still possible to get a good description of the orbits. To achieve this one associates with $D$ a distribution $\mathcal{T}(D)$, defined by letting, for each $m \in M, \mathcal{T}(D)(m)=$ linear hull of $D^{*}(m)$. Then Chow's theorem is applied to the maximal integral manifolds of $\mathcal{T}(D)$, and it follows that these manifolds are the orbits of $D$.

The method outlined in the preceding paragraph has an obvious drawback: the maximal integral manifolds of $\mathcal{T}(D)$ need not exist. This can be seen by simple examples (cf. $\$ 3$ ). However, in the se examples the orbits are still submanifolds, even though this fact cannot be proved by means of Chow's theorem. This suggests that the "pathology", when it occurs, arises from the integral manifolds, and not from the orbits. Our Theorem 4.1 substantiates this assertion. Roughly speaking, the orbits are always well behaved. Moreover, they are given as maximal integral manifolds of a $C^{\infty}$ distribution $P_{D}$. The reason why the method based on Chow's theorem is unable to handle the pathological cases is simply that, in general, the distribution $\mathcal{T}(D)$ does not coincide with $P_{D}$. The "right" distribution to look at is $P_{D}$, and the situations in which Chow's theorem can be applied correspond precisely to those cases when $P_{D}=\mathfrak{T}(D)$.

As a by-product of our work, we shall obtain a complete characterization of those $C^{\infty}$ distributions $\Delta$ that have the maximal integral manifolds property (i.e. through every point of $M$ there passes a maximal integral manifold of $\Delta$ ). This characterization is given in Theorem 4.2, and it implies all the known results about integrability of distributions. We shall now indicate how this result relates to previous work.

An obvious necessary condition for a $C^{\infty}$ distribution $\Delta$ to have integral manifolds is that it be involutive (the definition is given in $\$ 3$ ). If, in addition, the dimension of $\Delta(\mathrm{m})$ is constant, then this condition is also sufficient (this is the classical theorem of Frobenius, cf. Chevalley [1]). When the dimension of $\Delta(m)$ varies (i.e. when $\Delta$ has "singularities"), extra assumptions are needed. Hermann [4] stated various conditions that would guarantee integrability. Nagano [7] proved that integrability follows if $M$ is a real analytic manifold and $\Delta$ is an analytic distribution. Lobry [5] introduced a condition (that $\Delta$ be "locally of 
finite type") which also implies integrability. Moreover, since this condition is automatically satisfied in the analytic case, Lobry's work provides a new proof of Nagano's result. Matsuda [6] showed that integrability also follows from a "convergence condition'. Our Theorem 4.2 contains all these results. Its proof consists essentially of isolating from the conditions of Hermann and Lobry only what is strictly necessary to obtain integrability.

As a second by-product of our work, we submit that, by looking at orbits (rather than integral manifolds) as the fundamental objects to be studied, one obtains an approach that clarifies and unifies many known results. To substantiate this claim, we have made this paper practically self-contained, and included proofs (by our methods) of results that are not new. (Another reason for this is that the main applications of our results are to the study of controllability problems, and we wish to make this article readable by an audience which is only acquainted with the rudiments of differential geometry.) As an illustration of the advantages of our approach, we show in $\$ 7$ how our results imply Chow's theorem.

The organization of the paper is as follows: in $\$ 2$ we introduce the basic definitions and notations concerning families of vector fields, groups of local diffeomorphisms, and orbits. In particular, this section contains the definition of the "natural" topology of the orbits. In $\$ 3$ we define what is meant by a $C^{\infty}$ distribution, and we introduce the distribution $P_{D}$. A remark at the end the section explains its geometric meaning. In $\$ 4$ we state our two main theorems. The proofs of Theorems 4.1 and 4.2 are given in $\$ 5$ and $\$ 6$, respectively. In $\$ 7$ we derive Chow's theorem. In $\$ 8$ we discuss the connection of Theorem 4.2 with known results on integrability of distributions and, in particular, we derive the theorems of Frobenius, Hermann, Lobry and Matsuda. In $\$ 9$ we make a few remarks about the analytic case and indicate, following Lobry, how to derive Nagano's result.

Finally, two remarks on terminology.

(a) We have chosen to speak about "groups" of local diffeomorphisms to emphasize the analogy with the case of a Lie group acting on a manifold. These groups of local diffeomorphisms are not groups in the algebraic sense, unless they consist of everywhere defined diffeomorphisms. For a similar reason, we use the word "orbit" rather than "leaf" (Hermann [4], Lobry [5]). The reader who wishes to do so may substitute "pseudogroup" for "group" and/or "leaf " for "orbit".

(b) The distribution which we have denoted by $\mathcal{T}(D)$ (following [8]) will be denoted in the rest of this article by $\Delta_{D^{*}}$, according to the general notational conventions of $\$ 3$.

2. Families of vector fields and orbits. Throughout this paper, all manifolds are supposed to be of class $C^{\infty}$ and paracompact. If $M$ is a manifold, a submani- 
fold of $M$ is a manifold $S$ such that $S$ is a subset of $M$ and that the inclusion map from $S$ into $M$ is a $C^{\infty}$ map whose differential is everywhere injective (in particular, the inclusion map is continuous, but it is not required that $S$ be a topological subspace of $M$ ).

We shall use $M_{m}$ to denote the tangent space to the manifold $M$ at the point $m$. If $S$ is a submanifold of $M$, and if $s \in S, S_{s}$ can be identified in a natural way with a subspace of $M_{s}$. In particular, the meaning of expressions such as "the vector field $X$ is tangent to the submanifold $S$ " is clear.

$V(M)$ will denote the set of all $C^{\infty}$ vector fields defined on open subsets of $M$. If $X$ and $Y$ belong to $V(M)$, then the Lie bracket $[X, Y]$ of $X$ and $Y$ is a vector field which is defined on the intersection of the domains of definition of $X$ and $Y$, and which is given by the formula

$$
[X, Y] f=X(Y f)-Y(X f)
$$

for $f$ a $C^{\infty}$ real-valued function on $M$. (To avoid the need for remarks such as "provided the domains of $X$ and $Y$ intersect", we shall declare the empty vector field to be an element of $V(M)$. A similar remark applies to other concepts to be introduced later, such as local diffeomorphisms.)

If $X \in V(M)$, an integral curve of $X$ is a $C^{\infty}$ curve $t \rightarrow \gamma(t)$ such that, for every $t$ in the domain of definition of $\gamma$, the tangent vector to $\gamma$ at $t$ is $X(\gamma(t))$. By well-known theorems, for every $m$ in the domain of $X$ there exists an integral curve $\gamma$ of $X$ such that $\gamma(0)=m$, and which is defined in an open interval $I$ which contains the origin. Moreover, $\gamma$ is unique if we require, in addition, that the interval $I$ be maximal (i.e. if $\gamma$ is not the restriction of an integral curve which is defined on an interval which properly contains $l$ ). We shall refer to this curve as the integral curve of $X$ through $m$. To indicate the dependence on $X$ and $m$ (as well as $t$ ) we shall write $X_{t}(m)$ instead of $\gamma(t)$. It is well known that the mapping $(t, m) \rightarrow X_{t}(m)$ is a $C^{\infty}$ map from an open subset $\Omega(X)$ of $R \times M$ into $M$. For each $t, X_{t}$ is a diffeomorphism from an open subset $\Omega$ of $M$ onto an open subset $\Omega^{\prime}$. The set $\Omega$ (which may be empty) will be denoted by $\Omega_{t}(X)$. It is then clear that $\Omega^{\prime}$ is precisely $\Omega_{-t}(X)$.

We shall need some notations for composites of several maps of the form $X_{t}$. If $A$ is an arbitrary set, let $A^{m}$ denote the set of all (ordered) $m$-tuples of elements of $A$. Let $\xi \in V(M)^{m}$ and $T \in R^{m}, m \in M$. If $\xi=\left(X^{1}, \ldots, X^{m}\right)$ and $T=$ $\left(t_{1}, \cdots, t_{n}\right)$, then $\xi_{T}(m)$ will denote the point

$$
X_{t_{1}}^{1}\left(X_{t_{2}}^{2}\left(\cdots X_{t_{n}}^{n}(m) \cdots\right)\right) .
$$

It is clear that $\xi_{T}(m)$ will be defined for all $(T, m)$ which belong to an open subset of $R^{m} \times M$. We shall use $\Omega(\xi)$ to denote this set, and $\Omega_{T}(\xi)$ to denote 
the set of all $m$ such that $(T, m) \in \Omega(\xi)$ (i.e. the set of all $m$ for which $\xi_{T}(m)$ is defined).

A local diffeomorphism on $M$ is a $C^{\infty}$ diffeomorphism from an open subset $\Omega$ of $M$ onto an open subset $\Omega^{\prime}$. If $\delta_{i}: \Omega_{i} \rightarrow \Omega_{i}^{\prime}(i=1,2)$ are local diffeomorphisms, then the composite $\delta_{1} \delta_{2}$ is a local diffeomorphism, with domain $\delta_{2}^{-1}\left(\Omega_{1}\right)$ and range $\delta_{1}\left(\Omega_{2}^{\prime} \cap \Omega_{1}\right)$. The inverse of $\delta_{1}$ is denoted by $\delta_{1}^{-1}$, and is a local diffeomorphism with domain $\Omega_{1}^{\prime}$ and range $\Omega_{1}$. The formal laws

$$
\left(\delta_{1} \delta_{2}\right) \delta_{3}=\delta_{1}\left(\delta_{2} \delta_{3}\right) \text { and }\left(\delta_{1} \delta_{2}\right)^{-1}=\delta_{2}^{-1} \delta_{1}^{-1}
$$

are clearly valid.

A group of local diffeomorphisms is a set $G$ of local diffeomorphisms which is closed under compositions and inverses. If $X \in V(M)$ then the mapping $X_{t}$ is a local diffeomorphism for every $t$. The set of all such local diffeomorphisms is called the group of local diffeomorphisms generated by $X$, and is denoted by $G_{X}$. More generally, let $D$ be a subset of $V(M)$. There exists a smallest group of local diffeomorphisms which contains the union of the $G_{X}$ for $X \in D$. We shall use $G_{D}$ to denote this group, and we shall call it the group of local diffeomorphisms generated by $D$. It is clear that the elements of $G_{D}$ are precisely the mappings which are of the form $\xi_{T}$ where, for some positive integer $n, \xi \in D^{n}$ and $T \in R^{n}$.

If $\lambda=\left(\lambda_{1}, \ldots, \lambda_{n}\right)$ and $\mu=\left(\mu_{1}, \ldots, \mu_{r}\right)$ are finite sequences, we use $\lambda \mu$ to denote the sequence $\left(\lambda_{1}, \ldots, \lambda_{n}, \mu_{1}, \ldots, \mu_{r}\right)$. Also, $\hat{\lambda}$ will denote the sequence $\left(\lambda_{n}, \ldots, \lambda_{1}\right)$. With this notation, the operations of $G_{D}$ are given by:

$$
\xi_{T} \eta_{T^{\prime}}=(\xi \eta)_{T T^{\prime}} \text { and }\left(\xi_{T}\right)^{-1}=\xi_{-} .
$$

We say that a subset $D$ of $V(M)$ is everywbere defined if the union of the domains of the elements of $D$ is $M$. Similarly, a group of local diffeomorphisms $G$ is everywhere defined if every $m \in M$ belongs to the domain of some $g \in G$.

Let $G$ be an everywhere defined group of local diffeomorphisms on $M$. We say that two elements $m$ and $m^{\prime}$ of $M$ are G-equivalent if there is a $g \in G$ such that $g\left(m_{1}\right)=m_{2}$. This clearly defines an equivalence relation on $M$. The equivalence classes modulo this relation are called the orbits of $G$ (or $G$-orbits). If $D \subseteq V(M)$ is everywhere defined, the $G_{D}$-orbits will be referred to as the orbits of $D$, or $D$-orbits. Two points $m_{1}$ and $m_{2}$ belong to the same orbit if and only if, for some positive integer $n$, there exist $\xi \in D^{n}$ and $T \in R^{n}$ such that $\xi_{T}\left(m_{1}\right)=$ $m_{2}$. Equivalently, $m_{1}$ and $m_{2}$ belong to the same orbit if and only if there exists a curve $\gamma:[a, b] \rightarrow M$ such that $\gamma(a)=m_{1}, \gamma(b)=m_{2}$, and which has the following property:

(PI) There exist numbers $t_{i}$ such that $a=t_{0}<t_{1}<\cdots<t_{r}=b$ and vector fields $X^{i} \in D(i=1, \ldots, r)$ such that, for each $i$, the restriction of $\gamma$ to 
$\left[t_{i-1}, t_{1}\right]$ is an integral curve of $X^{i}$ or of $-X^{i}$.

A curve $\gamma$ which satisfies condition (PI) will be called a piecewise integral curve of $D$.

The orbits of $D$ can be given a natural topology. If $m \in M$ and $\xi \in D^{n}$, let $\rho_{\xi, m}$ denote the map $T \rightarrow \xi_{T}(m)$ and let $\Omega_{\xi, m}\left(\subseteq R^{n}\right)$ be its domain. Now let $S$ be the orbit of $D$ through $m$. Then $S$ is the union of the images of all the mappings $\rho_{\xi, m}$. We topologize $S$ by the strongest topology which makes all the $\rho_{\xi, m}$ (for all $n$ and all $\xi \in D^{n}$ ) continuous. Since the topology of $S$ as a subspace of $M$ has this property, it follows that the inclusion map from $S$ into $M$ is continuous. In particular, $S$ is Hausdorff. $S$ will not be, in general, a topological subspace of M. Since all the sets $\Omega_{\xi, m}$ are connected it follows that $S$ is connected.

We now verify that the topology so defined on $S$ does not depend on the choice of $m \in S$. For each $m \in S$, let $S^{m}$ denote $S$ with the topology defined above by means of the maps $\rho_{\xi, m}$. It is sufficient to prove that the inclusion map $i: S^{m} \rightarrow S^{m^{\prime}}$ is continuous for every $m, m^{\prime}$. Let $\eta, T_{0}$ be such that $\eta_{T_{0}}\left(m^{\prime}\right)=m$. Then $\rho_{\xi, m}$ is the mapping $T \rightarrow \xi_{T} \eta_{T_{0}}\left(m^{\prime}\right)$, which is the composite of $\stackrel{0}{T} \rightarrow T T_{0}$ and $\rho_{\xi \eta, m^{\prime}}$. Since $\rho_{\xi \eta, m^{\prime}}$ is continuous into $S^{m^{\prime}}$, it follows that $\rho_{\xi, m}: \Omega_{\xi, m} \rightarrow S^{m^{\prime}}$ is continuous. Since this is true for all $\xi$, our conclusion follows.

3. Distributions. A distribution on a manifold $M$ is a mapping $\Delta$ which assigns, to every $m \in M$, a linear subspace $\Delta(m)$ of the tangent space $M_{m}$.

A set of vector fields is said to span the distribution $\Delta$ if, for every $m \in M$, $\Delta(m)$ is the linear hull of the vectors $X(m)$, where $X$ belongs to the given set. If $D \subseteq V(M)$ is everywhere defined, there is a distribution $\Delta$ which is spanned by $D$. This distribution will be denoted by $\Delta_{D}$. A distribution which is of the form $\Delta_{D}$ for some everywhere defined subset $D$ of $V(M)$ is called a $C^{\infty}$ distribution.

A vector field $X \in V(M)$ belongs to the distribution $\Delta$ if $X(m) \in \Delta(m)$ for every $m$ in the domain of $X$. We let $D_{\Delta}$ denote the set of all $X \in V(M)$ which belong to $\Delta$. It is clear that $\Delta$ is a $C^{\infty}$ distribution if and only if $\Delta$ is spanned by $D_{\Delta}$.

If $G$ is a group of local diffeomorphisms on $M$, then the distribution $\Delta$ is said to be $G$-invariant if $d g$ maps $\Delta(m)$ into $\Delta(g(m))$ for every $m \in M$ and every $g$ such that $m$ belongs to the domain of $g$. If $\Delta$ is $G$-invariant and if $g, m$ are as above, then $d g^{-1}$ maps $\Delta(g(m))$ into $\Delta(m)$. In particular, the dimension of $\Delta(m)$ is the same for all points $m$ which belong to a given $G$-orbit.

If $\Delta_{1}$ and $\Delta_{2}$ are distributions, we say that $\Delta_{1}$ is contained in $\Delta_{2}$ if $\Delta_{1}(\mathrm{~m})$ $\subseteq \Delta_{2}(m)$ for every $m$. If $\Delta$ is a distribution and $G$ is a group of local diffeomorphisms, then there is a smallest distribution $\Delta^{G}$ which contains $\Delta$ and is $G$-invariant. The space $\Delta^{G}(m)$ is the linear hull of all the vectors $v \in V_{m}$ such that $v \in \Delta(m)$ or $v=d g(w)$ where $g \in G$ and, for some $m^{\prime} \in M, m=g\left(m^{\prime}\right)$ and $w \in \Delta\left(m^{\prime}\right)$. 
If $\Delta$ is spanned by $D \subseteq V(M)$, then $\Delta^{G}$ is spanned by the union of $D$ and the set of all vector fields which are images of elements of $D$ under local diffeomorphisms that belong to $G$. In particular, if $\Delta$ is a $C^{\infty}$ distribution, then $\Delta^{G}$ is also $C^{\infty}$.

If $D \subseteq V(M)$, a distribution which is $G_{D}$-invariant is said to be $D$-invariant. The smallest $D$-invariant distribution which contains $\Delta$ is denoted by $\Delta^{D}$.

Let $D$ be an everywhere defined subset of $V(M)$. We are interested in the distribution $\Delta_{D}^{D}$, i.e. the smallest distribution which is $D$-invariant and contains $\Delta_{D}$. This distribution will be denoted by $P_{D^{*}}$. Clearly, $P_{D}$ is a $C^{\infty}$ distribution. It follows from the preceding remarks that the dimension of $P_{D}(m)$ depends only on the $D$-orbit of $m$. If $S$ is a $D$-orbit, and if $m \in S$, then the dimension of $P_{D}(m)$ is called the rank of the orbit $S$.

A set $D \subseteq V(M)$ is said to be involutive if, whenever $X \in D$ and $Y \in D$, it follows that $[X, Y]$ belongs to $D$. If $D \subseteq V(M)$ is arbitrary, then there exists a smallest involutive subset of $V(M)$ which contains $D$. This set will be denoted by $D^{*}$.

A $C^{\infty}$ distribution $\Delta$ is involutive if the set $D_{\Delta}$ is involutive. It will be shown below that, if $D \subseteq V(M)$ is everywhere defined, then $P_{D}$ is involutive. Since every $X \in D$ belongs to $P_{D}$, we have the inclusions

$$
\Delta_{D} \subseteq \Delta_{D^{*}} \subseteq P_{D}
$$

It is clear that the first inclusion in (3.1) may be proper. The following example shows that this is also possible for the second inclusion. Let $M$ be $R^{2}$, and let the coordinates be denoted by $x$ and $y$. Let $D$ consist of two vector fields $X_{1}$ and $X_{2}$. Precisely, let

$$
X_{1}=\partial / \partial x, \quad X_{2}=\phi \partial / \partial y
$$

where $\phi(x, y)=\psi(x)$, and $\psi$ is a $C^{\infty}$ function such that $\psi(x)=0$ for $x \leq 0$ and $\psi(x)>0$ for $x>0$. Clearly, $P_{D}(x, y)$ has dimension two if $x>0$. Since every point of $M$ can be joined to a point $(x, y)$ with $x>0$ by a piecewise integral curve of $D$, it follows that $P_{D}$ has dimension two everywhere. On the other hand, it is clear that $\Delta_{D^{*}}$ has dimension one whenever $x \leq 0$.

A submanifold $S$ of $M$ is said to be an integral submanifold (or integral manifold) of the distribution $\Delta$ if, for every $s \in S$, the tangent space $S_{s}$ is exactly $\Delta(s)$. A $C^{\infty}$ distribution $\Delta$ has the integral manifolds property if for every $m \in M$ there exists an integral manifold $S$ of $\Delta$ such that $m \in S$. If $\Delta$ has the integral manifolds property then a $C^{\infty}$ vector field $X$ belongs to $\Delta$ if and only if $X$ is tangent to every integral manifold of $\Delta$. It follows that a $C^{\infty}$ distribution which bas the integral manifolds property must be involutive. The converse is not true, as shown by the distribution $\Delta_{D}$, where $D=\left\{X_{1}, X_{2}\right\}$ is the set of vector fields that was introduced in the example discussed above. 
If $\Delta$ is a $C^{\infty}$ distribution, a maximal integral manifold of $\Delta$ is a connected submanifold $S$ of $M$ such that

(a) $S$ is an integral manifold of $\Delta$, and

(b) every connected integral manifold of $\Delta$ which intersects $S$ is an open submanifold of $\Delta$.

It follows trivially from the definition that any two maximal integral submanifolds through a point $m$ must coincide.

We say that $\Delta$ has the maximal integral manifolds property if through every point $m \in M$ there passes a maximal integral manifold of $\Delta$.

Remark. The following considerations have the purpose of clarifying the geometric meaning of $\Delta_{D^{*}}$ and $P_{D}$. Suppose that we are trying to define a distribution $\Delta$ with the property that the orbits of $D$ are the maximal integral manifolds of $\Delta$. It is reasonable to define $\Delta(m)$ as the set of all tangent vectors at $m$ of curves $\gamma$ that pass through $m$ and are entirely contained in the $D$-orbit of $m$. Let $\Gamma_{m}$ be the set of all such curves. If $X \in D$, then the curve $t \rightarrow X_{t}(m)$ belongs to $\Gamma_{m}$. Therefore, $\Delta(m)$ must contain $X(m)$. Now let $X \in D, Y \in D$. The curve

$$
t \rightarrow X_{t}\left(Y_{t}\left(X_{-t}\left(Y_{-t}(m)\right)\right)\right)
$$

also belongs to $\Gamma$. After a reparametrization, it is well known that the tangent vector to this curve at $t=0$ is $[X, Y](m)$ (cf. Helgason [3, p. 97]). Therefore $\Delta(m)$ must also contain $[X, Y](m)$. A similar argument can be applied to higher brackets, and we conclude that $\Delta_{D^{*}}$ must be contained in $\Delta$. However, there may be more directions that have to be included in $\Delta(\mathrm{m})$, besides those of $\Delta_{D^{*}}(\mathrm{~m})$. For instance, let $X \in D$ and let $T \in R$ be fixed. Write $m^{\prime}=X_{-T}(m)$. If $t \rightarrow \gamma(t)$ is a curve such that $\gamma(0)=m^{\prime}$ and $\gamma \in \Gamma_{m^{\prime}}$, then the curve $\delta$ given by $t \rightarrow$ $X_{T}(\gamma(t))$ belongs to $\Gamma_{m}$. If $v$ is the tangent vector to $\gamma$ at $t=0$, then the tangent to $\delta$ at $t=0$ is $d X_{T}(v)$. Therefore, if $v \in \Delta\left(m^{\prime}\right)$, then $d X_{T}(v)$ must belong to $\Delta(m)$. Therefore, $\Delta$ must be $D$-invariant. This suggests that we define $\Delta$ to be the smallest $D$-invariant distribution which contains $\Delta_{D^{*}}$. It would not be hard to prove directly that the same result is achieved by taking $\Delta$ to be the smallest $D$-invariant distribution that contains $\Delta_{D}$, i.e. by taking $\Delta=P_{D}$ (this will follow from the results of the next section. Specifically, the inclusion $\Delta_{D^{*}} \subseteq P_{D}$ is a corollary of Theorem 4.1). The reason why $\Delta_{D^{*}}$ may not be the "right" distribution to look at can now be understood: It may happen that $\Delta_{D^{*}}$ does not contain sufficiently many directions. More precisely, one may move within the orbit of $m$ by "going far away", moving along an integral curve of an $X \in D$, and then coming back. Directions of motion obtained in this way need not belong to $\Delta_{D^{*}}(m)$, but they will belong to $P_{D}(m)$. 
4. Statement of the main results. We now state the two main theorems.

Theorem 4.1. Let $M$ be a $C^{\infty}$ manifold, and let $D$ be an everywhere defined set of $C^{\infty}$ vector fields. Then

(a) If $S$ is an orbit of $D$, then $S$ (with the topology of \$2) admits a unique differentiable structure such that $S$ is a submanifold of $M$. The dimension of $S$ is equal to its rank, as defined in $\$ 3$.

(b) With the topology and differentiable structure of (a), every orbit of $D$ is a maximal integral submanifold of $P_{D}$.

(c) $P_{D}$ bas the maximal integral manifolds property.

(d) $P_{D}$ is involutive.

Our formulation of Theorem 4.1 was chosen so that the statement will contain all the relevant information about the orbits of $D$ and the distribution $P_{D}$. Notice that parts (c) and (d) of Theorem 4.1 are immediate corollaries of (a) and (b).

We now turn to our second theorem.

Theorem 4.2. Let $M$ be a $C^{\infty}$ manifold, and let $\Delta$ be a $C^{\infty}$ distribution on M. Let $D$ be a set of $C^{\infty}$ vector fields which spans $\Delta$ (so that, in particular, $D$ is everywhere defined). Then the following conditions are equivalent:

(a) $\Delta$ bas the integral manifolds property.

(b) $\Delta$ bas the maximal integral manifolds property.

(c) $\Delta$ is D-invariant.

(d) For every $X \in D, t \in R, m \in M$ such that $X_{t}(m)$ is defined, then $d X_{t}$ maps $\Delta(m)$ into $\Delta\left(X_{t}(m)\right)$.

(e) For every $m \in M$ there exist elements $X^{1}, \ldots, X^{k}$ of $D$ such that

(1) $\Delta(m)$ is the linear bull of $X^{1}(m), \ldots, X^{k}(m)$, and

(2) for every $X \in D$ there exists $\epsilon>0$ such that there are $C^{\infty}$ functions $f_{j}^{i}(1 \leq i, j \leq k)$ which are defined in the open.interval $(-\epsilon, \epsilon)$ and satisfy

$$
\left[X, X^{i}\right]\left(X_{t}(m)\right)=\sum_{j=1}^{k} f_{j}^{i}(t) X^{j}\left(X_{t}(m)\right)
$$

for $-\epsilon<t<\epsilon, i=1, \cdots, k$.

(f) $\Delta=P_{D}$.

Theorems 4.1 and 4.2 will be proved in the following two sections.

5. Proof of Theorem 4.1. We let $D$ be an everywhere defined set of $C^{\infty}$ vector fields on $M$. Let $S$ be an orbit of $D$. We give $S$ the topology of $\$ 2$. Let $k$ denote the rank of $S$. We shall use the notations $\rho_{\xi, m}, \Omega_{\xi, m}$ that were introduced in $\$ 2$. Let $D^{\infty}$ denote the union of the sets $D^{n}$, taken over all positive integers $n$. Recall that, if $\xi \in D^{n}$, then $\Omega_{\xi, m}$ is an open subset of $R^{n}$, and 
$\rho_{\xi, m}$ is a $C^{\infty}$ map from $\Omega_{\xi, m}$ into $M$. Moreover, if $m \in S$ then $\rho_{\xi, m}$ is a continuous map into $S$. If $\xi \in D^{\infty}, m \in M, T \in \Omega_{\xi, m}$, we let $V(\xi, m, T)$ denote the image of the tangent space to $\Omega_{\xi, m}$ at $T$ under the differential of $\rho_{\xi, m}$. It follows that $V(\xi, m, T)$ is a subspace of $M_{m^{\prime}}$, where $m^{\prime}=\xi_{T}(m)$.

Theorem 4.1 will be a consequence of the following lemmas.

Lemma 5.1. Let $\xi \in D^{\infty}, m \in S, T \in \Omega_{\xi, m}, m_{0}=\xi_{T}(m)$. Then $V(\xi, m, T)$ is contained in $P_{D}\left(m_{0}\right)$.

Lemma 5. 2. Let $m_{0} \in S$. Then there exist $\xi \in D^{\infty}, m \in S, T \in \Omega_{\xi, m}$ sucb that $\xi_{T}(m)=m_{0}$ and $V(\xi, m, T)=P_{D}\left(m_{0}\right)$.

Lemma 5.3. Let $N$ be a connected integral submanifold of $P_{D}$ and let $U$ be its underlying set of points. If $U$ intersects $S$ then $U$ is an open subset of $S$.

Proof that the lemmas imply Theorem 4.1. Let $m_{0} \in S$. By Lemma 5.2 there exist $m \in S, \xi \in D^{\infty}, T \in \Omega_{\xi, m}$ such that $\xi_{T}(m)=m_{0}$ and $V(\xi, m, T)=P_{D}\left(m_{0}\right)$. The differential of $\rho_{\xi, m}$ has rank $k$ at $T$. By Lemma 5.1, the rank of $d \rho_{\xi, m}$ cannot exceed $k$ at any point of $\Omega_{\xi, m}$. Let $\xi \in D^{n}$. By a.well-known form of the implicit function theorem, there exist neighbourhoods $U$ of $T$ in $R^{n}$ and $V$ of $m_{0}$ in $M$, and diffeomorphisms $\phi, \psi$ from $U$ onto $C^{n}$ and from $V$ onto $C^{\mu}$ such that the following diagram

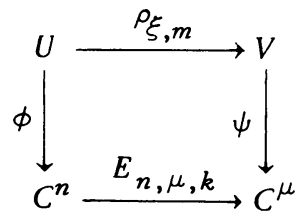

commutes, and that $\phi(T)=0, \psi\left(m_{0}\right)=0$. Here we are using the notations

$$
\begin{gathered}
C^{p}=\left\{\left(x_{1}, \cdots, x_{p}\right):-1<x_{i}<1 \text { for } i=1, \cdots, p\right\}, \\
E_{n, \mu, k}\left(x_{1}, \cdots, x_{n}\right)=\left(x_{1}, \cdots, x_{k}, 0, \cdots, 0\right),
\end{gathered}
$$

where " $0, \ldots, 0$ " denotes a string of $\mu-k$ zeroes, and $\mu=\operatorname{dim} M$.

Let $\Lambda$ denote the submanifold of $M$ which is the inverse image under $\psi$ of $E_{n, \mu, k}\left(C^{n}\right)$. The set of points of $\Lambda$ is precisely $\rho_{\xi, m}(U)$. If $T^{\prime} \in U$, then the tangent space of $\Lambda$ at $m^{\prime}=\rho_{\xi, m}\left(T^{\prime}\right)$ is $V\left(\xi, m, T^{\prime}\right)$. By Lemma 5.1, $V\left(\xi, m, T^{\prime}\right)$ is contained in $P_{D}\left(m^{\prime}\right)$. Since both spaces have dimension $k$, it follows that $\Lambda_{m^{\prime}}=P_{D^{\prime}}\left(m^{\prime}\right)$. Therefore $\Lambda$ is an integral submanifold of $P_{D^{*}}$. It is clear that $\Lambda$ is contained in $S$. By Lemma 5.3, the set of points of $\Lambda$ is open in $S$. Let $I$ denote the inclusion map from $\Lambda$ into $S$. Lemma 5.3 can be applied to every open connected subset $W$ of $\Lambda$. Moreover, the open connected sets constitute a basis 
for the topology of $\Lambda$. Therefore, $I$ is an open map. Moreover, $I$ is the composite of the inclusion $I^{\prime}: \Lambda \rightarrow V$, the diffeomorphism $\psi$, the mapping $E_{\mu, n, k}$, the diffeomorphism $\phi^{-1}$ and the map $\rho_{\xi, m}$. Since $\rho_{\xi, m}$ is continuous as a map into $S$, we conclude that $I$ is continuous. Therefore we have shown that $I$ is a homeomorphism onto its image and that this image is open in $S$.

Let $\Sigma$ denote the set of all manifolds $\Lambda$ that can be obtained by the construction that was described above. For each $\Lambda \in \Sigma$, let $U(\Lambda)$ denote the underlying set of $\Lambda$. It is clear that the sets $U(\Lambda)$ form an open covering of $S$, and that for each $\Lambda \in \Sigma$ the inclusion of $\Lambda$ onto $S$ is a homeomorphism onto its image. Therefore, we have a family of differentiable structures on a family of open subsets of $S$ which cover $S$. We want to define a differentiable structure on $S$ in such a way that all the members of $\Sigma$ will be open submanifolds of $S$. It is sufficient to prove that, if $\Lambda_{i} \in \Sigma(i=1,2)$ then the differentiable structures of $U\left(\Lambda_{1}\right) \cap U\left(\Lambda_{2}\right)$ as open submanifolds of $\Lambda_{1}$ and $\Lambda_{2}$ coincide. Let these manifolds be denoted by $W_{1}, W_{2}$, and let $J: W_{1} \rightarrow W_{2}$ be the identity map. It is sufficient to prove that $J$ is $C^{\infty}$. Since $W_{2}$ is a submanifold of $M$, this will follow if we show that $J$ is continuous. But the continuity of $J$ is a consequence of the fact that the inclusions of $W_{i}$ into $S$ are homeomorphisms for $i=1,2$.

We have proved that $S$ admits a differentiable structure which is compatible with the topology of $\$ 2$, and is such that $S$ is a submanifold of $M$. The uniqueness of such a structure is immediate. It follows from our construction that $S$ is a $k$-dimensional manifold, and that $S$ is an integral submanifold of $P_{D}$.

We now show that $S$ is a maximal integral subman ifold of $P_{D}$. It is clear that $S$ is connected (cf. $\$ 2$ ). Let $\Gamma$ be a connected integral submanifold of $P_{D}$ which intersects $S$. By Lemma 5.3, the set $U(\Gamma)$ of points of $\Gamma$ is an open subset of $S$. As before, we can apply Lemma 5.3 to every open connected subset of $\Gamma$, and use the fact that these sets are a basis for the topology of $\Gamma$. It follows that the inclusion map from $\Gamma$ into $S$ is open. Let $\Gamma^{\prime}$ be the open submanifold of $S$ whose underlying set is $U(\Gamma)$. Let $I: \Gamma^{\prime} \rightarrow \Gamma$ be the identity map. The preceding remarks show that $I$ is continuous. Since $\Gamma^{\prime}$ and $\Gamma$ are submanifolds of $M$, it follows that $I$ is $C^{\infty}$. Since $I$ is regular, we conclude that $I$ is a $C^{\infty}$ diffeomorphism. Therefore $\Gamma=\Gamma^{\prime}$, so that $\Gamma$ is an open submanifold of $S$. The proof that $S$ is a maximal integral submanifold of $P_{D}$ is complete.

The preceding paragraphs establish statements (a) and (b) of Theorem 4.1. The remaining statements follow trivially from these. The proof of Theorem 4.1 is now complete, modulo Lemmas 5.1, 5.2 and 5.3.

Proof of Lemma 5.1. Let $\xi \in D^{n}$. We prove our result by induction on $n$. If $n=1$, the desired conclusion reduces to the assertion that the tangent vector at $t_{0}$ to the curve $t \rightarrow X_{t}(m)$ belongs to $P_{D}\left(X_{t_{0}}(m)\right)$ for every $X \in D$ and every $t_{0}$ 
such that $X_{t_{0}}(m)$ is defined. The truth of this assertion is immediate given the definition of $P_{D}$.

Now let $\xi \in D^{n}, T \in D^{n}$. Write $\xi=X \eta, T=t_{0} T^{\prime}$, where $X \in D, \eta \in D^{n-1}$, $T^{\prime} \in \Omega_{\eta, m}, t_{0} \in R$. Assume that our conclusion is true for $\eta$. It is clear that $V(\xi, m, T)$ is spanned by $X\left(\xi_{T}(m)\right)$ and the image under $d X_{t_{0}}$ of $V\left(\eta, m, T^{\prime}\right)$. Since $V\left(\eta, m, T^{\prime}\right)$ is contained in $P_{D}\left(\eta_{T},(m)\right)$, and $P_{D}$ is $D$-invariant, it follows that

$$
d X_{t_{0}}\left(V\left(\eta, m, T^{\prime}\right)\right) \subseteq P_{D}\left(\xi_{T}(m)\right)
$$

Also, it is clear that $X\left(\xi_{T}(m)\right)$ belongs to $P_{D}\left(\xi_{T}(m)\right)$. Therefore $V(\xi, m, T)$ is contained in $P_{D}\left(\xi_{T}(m)\right)$. The proof of Lemma 5.1 is now complete.

Proof of Lemma 5.2. We shall prove the following two assertions:

(a) If $\xi \in D^{\infty}, m \in S, T \in \Omega_{\xi, m}, \eta \in D^{\infty}, m^{\prime} \in S, T^{\prime} \in \Omega_{\eta, m^{\prime}}$, are such that $\xi_{T}(m)=\eta_{T}{ }^{\prime}\left(m^{\prime}\right)=m_{0}$, then there exist $\sigma \in D^{\infty}, m^{\prime \prime} \in S, T^{\prime \prime} \in \Omega_{\sigma, m^{\prime \prime}}$ such that $V(\xi, m, T)$ and $V\left(\eta, m^{\prime}, T^{\prime}\right)$ are contained in $V\left(\sigma, m^{\prime \prime}, T^{\prime \prime}\right)$.

(b) There is a subset $A$ of $P_{D}\left(m_{0}\right)$ which spans $P_{D}\left(m_{0}\right)$ and is such that for every $v \in A$ there exist $\xi \in D^{\infty}, m \in S, T \in \Omega_{\xi, m}$ such that $\xi_{T}(m)=m_{0}$ and $v \in V(\xi, m, T)$.

It is clear that Lemma 5.2 follows from (a), (b) and Lemma 5.1. Therefore, we only have to prove (a) and (b).

To prove (a), take $m^{\prime \prime}=m^{\prime}, \sigma=\xi \hat{\xi} \eta, T^{\prime \prime}=T(-\hat{T}) T^{\prime}$ (here we use the notations of $\$ 2)$. Then $\sigma_{T^{\prime \prime}}\left(m^{\prime \prime}\right)=\xi_{T}\left(\hat{\xi}_{-T}\left(\eta_{T^{\prime}}\left(m^{\prime}\right)\right)\right)=\eta_{T^{\prime}}\left(m^{\prime}\right)=m_{0}$. Since $\xi_{T} \hat{\xi}_{-T}$ is the identity mapping, it is clear that $V\left(\sigma, m^{\prime \prime}, T^{\prime \prime}\right)$ will contain $V\left(\eta, m^{\prime}, T^{\prime}\right)$. Moreover, it is immediate from the definition of $\sigma, T^{\prime \prime}$, and $m^{\prime \prime}$ that $V\left(\sigma, m^{\prime \prime}, T^{\prime \prime}\right)$ also contains $V(\xi, m, T)$. This proves (a).

To prove (b), we take $A$ to be the set of all vectors $X\left(m_{0}\right)$, where $X$ is the vector field which corresponds to some $Y \in D$ under a local diffeomorphism $g \in G_{D}$. It is clear that $A$ spans $P_{D}\left(m_{0}\right)$. Let $v \in A$, and let $v=d g(w)$, where $g \in G_{D}$ and, for some $m \in S, g(m)=m_{0}$ and $w \in M_{m}$ is of the form $X(m), X \in D$. Let $g=\xi_{T}\left(\xi \in D^{\infty}, T \in \Omega_{\xi, m}\right)$. Let $\eta=\xi X, T^{\prime}=(T, 0)$. Then $\eta_{T^{\prime}}(m)=\xi_{T}(m)=m_{0}$. Moreover, the image of the differential of $\rho_{\eta, m}$ at $T^{\prime}$ certainly contains $d \xi_{T}(X(m))$. Therefore, $v \in V\left(\eta, m, T^{\prime}\right)$, and (b) is proved. The proof of Lemma 5.2 is now complete.

Proof of Lemma 5.3. Let $D$ be the set of all vector fields that are of the form $d g(X)$, for some $X \in D, g \in G_{D}$. Let $Y \in \mathcal{D}$. Let $Y=d g(X), g \in G_{D}, X \in D$. If $\gamma$ is an integral curve of $Y$, then $\gamma$ is the image under $g$ of an integral curve of $X$. From this it is clear that any two points in $\gamma$ are in the same $D$-orbit.

Let $N$ be a connected integral submanifold of $P_{D}$, and let $m \in N$. Let $X^{1}$, $\cdots, X^{p}$ be elements of $\mathscr{D}$ such that $\left\{X^{1}(m), \cdots, X^{p}(m)\right\}$ is a basis for $P_{D}(m)$. 
The mapping

$$
\left(t_{1}, \cdots, t_{p}\right) \rightarrow X_{t_{1}}^{1}\left(X_{t_{2}}^{2}\left(\ldots X_{t_{p}}^{p}(m) \ldots\right)\right)
$$

is a diffeomorphism from a neighborhood of 0 in $R^{p}$ onto a neighborhood of $m$ in $N$. The preceding remarks imply that every point in the image of this mapping belongs to the same orbit as $m$. We have shown that every point of $N$ has a neighborhood which is entirely contained in one orbit of $D$. Since $N$ is connected, we conclude that the set $U$ of points of $N$ is contained in an orbit of $D$. If $U \cap S$ is nonempty, it follows that $U \subseteq S$.

We now prove that $U$ is open in $S$. We must show that, if $m \in S, \xi \in D^{n}$, then $\rho_{\xi, m}^{-1}(U)$ is an open subset of $R^{n}$. Let $T \in \Omega_{\xi, m}\left(\subseteq R^{n}\right)$ be such that $\rho_{\xi, m}(T)$ $\in U$. It is clear that, for each $i$ such that $1 \leq i \leq n$, the image under $\rho_{\dot{\xi}, m}$ of any curve $t \rightarrow\left(\tau_{1}, \cdots, \tau_{i-1}, t, \tau_{i+1}, \cdots, \tau_{n}\right)$ is an integral curve of an $X \in \mathcal{D}$. If $\gamma$ is one such image, it follows that, if $\gamma\left(t_{0}\right) \in U$ for some $t_{0}$, then $\gamma(t) \in U$ for all $t$ in some neighborhood of $t_{0}$. From this it follows easily that the image under $\rho_{\xi, m}$ of some neighborhood of $T$ is contained in $U$. Therefore $\rho_{\xi, m}^{-1}(U)$ is open, and Lemma 5.3 is proved.

6. Proof of Theorem 4.2. The implications (d) $\Rightarrow(c) \Rightarrow(f)$ and (b) $\Rightarrow$ (a) are trivial. The implication $(f) \Rightarrow(b)$ follows from Theorem 4.1. We now show that (a) $\Rightarrow(\mathrm{e}) \Rightarrow(\mathrm{d})$. Assume that $\Delta$ has the integral manifolds property. Let $m \in M$ and let $S$ be an integral manifold of $\Delta$ through $m$. Let $X^{1}, \ldots, X^{k}$ be elements of $D$ such that $X^{1}(m), \cdots, X^{k}(m)$ form a basis for $\Delta(m)$. If $X \in D$ then the vector fields $\left[X, X^{i}\right]$ are tangent to $S$. Therefore their restrictions to $S$ are linear combinations of the $X^{i}$ with smooth coefficients, in some neighborhood $U$ of $\mathrm{m}$ (in $S$ ). If $\epsilon>0$ is small enough, then the curve $t \rightarrow X_{t}(m)$ is contained in $U$ for $|t|<\epsilon$. From this it is immediate that (a) $\Rightarrow(\mathrm{e})$.

We now show that $(e) \Rightarrow(d)$. The following lemma is a trivial consequence of the definition of Lie bracket, but we state it separately because we shall need it again later.

Lemma 6.1. Let $X$ and $Y$ be $C^{\infty}$ vector fields on $M$. Let $m \in M$, and let $\epsilon>0$ be such that $X_{t}(m)$ is defined for $|t|<\epsilon$. Let

$$
W(t)=d X_{-t}\left(Y\left(X_{t}(m)\right)\right) \text { for }|t|<\epsilon,
$$

so that $W(t) \in M_{m}$. Then $W(t)$ satisfies the differential equation

$$
W^{\prime}(t)=d X_{-t}\left([X, Y]\left(X_{t}(m)\right)\right)
$$

with initial condition $W(0)=Y(m)$. 
Now assume that condition (e) holds. To prove (d), it is sufficient to do it for $|t|<\epsilon, \epsilon>0$. Let $X^{1}, \cdots, X^{k}, \epsilon,\left(f_{j}^{i}\right)_{i, j=1, \cdots, k}$ be given by condition (e). Let $W^{i}(t) \in M_{m}$ be defined by

$$
W^{i}(t)=d X_{-t}\left(X^{i}\left(X_{t}(m)\right)\right) \text { for }|t|<\epsilon .
$$

By Lemma 6.1, we have

$$
d W^{i}(t) / d t=d X_{-t}\left(\left[X, X^{i}\right]\left(X_{t}(m)\right)\right) .
$$

The right side of this equation is equal to

$$
\sum_{j=1}^{k} f_{j}^{i}(t) d X_{-t}\left(X^{j}\left(X_{t}(m)\right)\right) .
$$

Therefore, the $W^{i}$ satisfy the system of differential equations

$$
\frac{d W^{i}}{d t}=\sum_{j=1}^{k} f_{j}^{i} W^{j}
$$

Since $W^{1}(0), \cdots, W^{k}(0)$ are a basis for $\Delta(m)$, it follows that $W^{1}(t), \cdots$, $W^{k}(t)$ form a basis for $\Delta(m)$ for $-\epsilon<t<\epsilon$. Since

$$
d X_{t}\left(W^{i}(t)\right)=X^{i}\left(X_{t}(m)\right) \in \Delta\left(X_{t}(m)\right),
$$

we conclude that $d X_{t}$ maps $\Delta(m)$ into $\Delta\left(X_{t}(m)\right)$, and our proof is complete.

Remark. The preceding proof is essentially contained in Lobry [5].

7. A theorem of Chow. An everywhere defined set $D$ of $C^{\infty}$ vector fields on a manifold $M$ is said to satisfy the reachability condition if the $D$-orbits are exactly the connected components of $M$. The following theorem gives a necessary and sufficient condition for reachability.

Theorem 7.1. Let $D$ be an everywhere defined set of $C^{\infty}$ vector fields on the n-dimensional $C^{\infty}$. manifold $M$. Then $D$ satisfies the reachability condition if and only if $P_{D}(m)$ bas dimension $n$ for every $m \in M$.

Proof. If $\operatorname{dim} P_{D}(m)=n$ for every $m \in M$, then the maximal integral submanifolds of $P_{D}$ are precisely the connected components of $M$. By (b) of Theorem 4.1, $D$ satisfies the reachability condition. Conversely, suppose that the dimension $k$ of $P_{D}(m)$ is less than $n$ for some $m \in M$. Then the orbit $S$ of $D$ through $m$ is a $k$-dimensional connected submanifold of $M$, and $k<n$. Therefore the $M$-interior of $S$ is empty. Then the reachability condition is not satisfied.

Corollary 7.2. Let $M, n, D$ be as in Theorem 7.1. Let $\Delta_{D^{*}}$ be the distribu- 
tion spanned by $D^{*}$. If $\Delta_{D^{*}}(m)$ bas dimension $n$ for every $m \in M$, then $D$ satis. fies the reachability condition.

Proof. Every $X \in D$ belongs to $P_{D}$. Since $P_{D}$ is involutive, it follows that every $X \in D^{*}$ belongs to $P_{D}$. Therefore $\Delta_{D^{*}} \subseteq P_{D}$. Our assumption then implies that $P_{D}(m)$ has dimension $n$ for every $m \in M$. The conclusion now follows from Theorem 7.1.

The preceding corollary is due to Chow [2]. We emphasize that the sufficient condition for reachability given in this result is by no means necessary. A simple example is obtained by considering the pair $\left\{X_{1}, X_{2}\right\}$ of vector fields in the plane that was introduced in $\$ 3$. In this example, however, there are points $m$ such that $\Delta_{D^{*}}(m)$ has maximal dimension. The following slight modification of the example shows that it is possible to have reachability even when $\Delta_{D^{*}}(m)$ does not have maximal dimension at any point $m \in M$. We take $M=R^{3}$, and we let the set $D$ consist of three vector fields $X_{1}, X_{2}, X_{3}$, which are defined as follows:

$$
X_{1}=\partial / \partial x, \quad X_{2}=\phi \partial / \partial y, \quad X_{3}=\psi \partial / \partial z
$$

Here $\phi(x, y, z)=\rho(x)$ and $\psi(x, y, z)=\sigma(x)$. The functions $\rho$ and $\sigma$ are infinitely differentiable and satisfy

$$
\begin{aligned}
& \rho(x)=0 \text { for } x \leq 0, \quad \sigma(x)=0 \text { for } x \geq-1 \text {, } \\
& \rho(x)>0 \text { for } x>0, \quad \sigma(x)>0 \text { for } x<-1 .
\end{aligned}
$$

One sees easily that the reachability condition is satisfied. However, the dimension of $\Delta_{D^{*}}(x, y, z)$ never exceeds two.

Theorem 7.1 and the preceding example justify the claim made in the introduction that, in general, $\Delta_{D^{*}}$ is not the "right" distribution that is needed for the study of $D$-orbits.

8. Integrability of distributions. We now discuss various conditions under which it has been proved that a distribution has integral submanifolds. Our purpose is to show how the known results follow from ours.

An everywhere defined set $D$ of $C^{\infty}$ vector fields is said to be locally of finite type if for every $m \in M$ there exist vector fields $X^{1}, \ldots, X^{k}$ which belong to $D$ and satisfy

(LFT1) $X^{1}(m), \ldots, X^{k}(m)$ span $\Delta_{D}(m)$, and

(LFT2) for every $X \in D$ there exist a neighborhood $U$ of $m$ and $C^{\infty}$ functions $f_{j}^{i}$, defined on $U$, which satisfy

$$
\left[X, X^{i}\right]\left(m^{\prime}\right)=\sum_{j=1}^{k} f_{j}^{i}\left(m^{\prime}\right) X^{j}\left(m^{\prime}\right)
$$

for all $m^{\prime} \in U$. 
Theorem 8.1. If $D$ is locally of finite type then $\Delta_{D}$ bas the maximal integral manifolds property.

Proof. If $D$ is locally of finite type then it is clear that condition (e) of Theorem 4.2 is satisfied (with $\Delta=\Delta_{D}$ ). Therefore, our conclusion follows from the equivalence of (e) with condition (a) of the same theorem.

The preceding result is due to Hermann [4] and Lobry [5]. If $D \subseteq V(M)$ is such that $D^{*}$ is locally of finite type, it follows that $\Delta_{D^{*}}$ has the maximal integral manifolds property. By Theorems 4.1 and $4.2, \Delta_{D^{*}}=P_{D}$ and the $D$-orbits are precisely the maximal integral manifolds of $\Delta_{D^{*}}$.

The classical theorem of Frobenius can now be proved easily.

Theorem 8.2. Let $\Delta$ be a $C^{\infty}$ distibution on $M$ such that the dimension $k$ of $\Delta(m)$ is independent of $m$. Then $\Delta$ bas the maximal integral manifolds property if and only if it is involutive.

Proof. We know that if $\Delta$ has the maximal integral manifolds property then $\Delta$ is involutive. Conversely, let us assume that $\Delta$ is involutive. Let $m \in M$. Let $X^{1}, \ldots, X^{k}$ be $k C^{\infty}$ vector fields which belong to $\Delta$ and are such that $X^{1}(m)$, $\ldots, X^{k}(m)$ form a basis for $\Delta(m)$. Clearly, we can assume that $X^{1}\left(m^{\prime}\right), \ldots$, $X^{k}\left(m^{\prime}\right)$ are linearly independent for all $m^{\prime}$ in a neighborhood of $m$. Since the dimension of $\Delta\left(m^{\prime}\right)$ is always $k$, it follows that, in some neighborhood of $m$, every vector field that belongs to $\Delta$ is a linear combination of $X^{1}, \ldots, X^{k}$ with smooth coefficients. If $X$ belongs to $\Delta$, the preceding conclusion applies in particular to the vector fields $\left[X, X^{i}\right]$, which belong to $\Delta$ because $\Delta$ is involutive. We have shown that $\Delta$ is locally of finite type. The conclusion now follows from Theorem 8.1 .

In particular, the preceding result applies when $\Delta$ is the distribution spanned by an involutive set $D^{*}$ :

Corollary 8.3. Let $D^{*}$ be an everywhere defined set of $C^{\infty}$ vector fields. As. sume that $D^{*}$ is involutive and that $\Delta_{D^{*}}$ bas constant dimension $k$. Then $\Delta_{D^{*}}$ has the maximal integral manifolds property.

Proof. By Theorem 8.2, it will be sufficient to show that $\Delta_{D^{*}}$ is involutive. Let $m \in M$ and let $X^{1}, \ldots, X^{k}$ be elements of $D^{*}$ which are defined on a neighborhood $U$ of $m$ and are linearly independent at $m$. Let $X$ and $X^{\prime}$ belong to $\Delta_{D^{*}}$ near $m$. Then $X$ and $X^{\prime}$ are linear combinations of the $X^{i}$ with smooth coefficients. The formula

$$
[f Y, g Z]=(f \cdot Y g) Z-(g \cdot Z f) Y+f g[Y, Z]
$$

together with the fact that the brackets $\left[X^{i}, X^{j}\right]$ are elements of $D^{*}$, implies that $\left[X, X^{\prime}\right]$ belongs to $\Delta$. 
We now show that our results also contain those of Matsuda [6]. Matsuda considers a Lie algebra $L$ of (everywhere defined) $C^{\infty}$ vector fields on the manifold $M$. Let us define inductively

$$
\left[X^{(0)}, Y\right]=Y, \quad\left[X^{(k+1)}, Y\right]=\left[X,\left[X^{(k)}, Y\right]\right]
$$

for any two $C^{\infty}$ vector fields $X$ and $Y$ on $M$. Matsuda's condition is

(C) For every $X$ and $Y$ in $L$ and for every $m_{0} \in M$, the series

$$
g_{t}(X, Y)(m)=\sum_{k=0}^{\infty}(-1)^{k} \frac{t^{k}}{k !}\left[X^{(k)}, Y\right](m)
$$

converges for $(t, m)$ in some neighborhood of $\left(0, m_{0}\right)$. Moreover, $g_{t}(X, Y)(m)$ defines a continuously differentiable function of $t$ and $m$, and the derivatives of this function can be obtained by termwise differentiation of the series.

For each $m \in M$, let $L(m)=\{X(m): X \in L\}$. We prove:

Theorem 8.4. If the Lie algebra L satisfies Matsuda's condition (C), then the distribution $m \rightarrow L(m)$ bas the maximal integral manifolds property.

Proof. In view of Theorem 4.2, it is sufficient to prove that, if $X \in L$, $m_{0} \in M$, and $X_{t}\left(m_{0}\right)$ is defined, then

$$
d X_{t}\left(L\left(m_{0}\right)\right) \subseteq L\left(X_{t}\left(m_{0}\right)\right)
$$

Clearly, it is sufficient to show that, if $v \in L\left(m_{0}\right)$, then $d X_{t}(v)$ belongs to $L\left(X_{t}\left(m_{0}\right)\right)$ for sufficiently small $t$. Let $v=Y\left(m_{0}\right)$, where $Y \in L$. Let $\epsilon>0$ be such that

$$
V(t, \tau)=\sum_{k=0}^{\infty}(-1)^{k} \frac{t^{k}}{k !}\left[X^{(k)}, Y\right]\left(X_{\tau}\left(m_{0}\right)\right)
$$

converges for $|t|<\epsilon,|\tau|<\epsilon$, and can be differentiated term by term. Let $W(t, \tau)=$ $d X_{-\tau}(V(t, \tau))$ so that $W(t, \tau) \in M_{m_{0}}$. It follows from Lemma 6.1 that the derivative with respect to $\tau$ of $d X_{-\tau}\left(\left[X^{(k)}, Y\right]\left(X_{\tau}(m)\right)\right)$ is $d X_{-\tau}\left[X^{(k+1)}, Y\right]\left(X_{\tau}(m)\right)$. From this it follows immediately that $\partial W / \partial t+\partial W / \partial r=0$.

In particular, the function $t \rightarrow W(t, t)$ is a constant, so that $W(t, t)=v$ for all $t$.

Therefore, $d X_{t}(v)=V(t, t)$. Since $L$ is a Lie algebra of vector fields, it is clear that $V(t, \tau)$ belongs to $L\left(X_{\tau}(m)\right)$ for every $t, \pi$. Therefore $d X_{t}(v) \in L\left(X_{t}(m)\right)$, and our theorem is proved.

9. The analytic case. If $M$ is a real analytic manifold, then all the definitions of $\$ 2$ and $\$ 3$ can be reformulated with " $C$ " replaced by "analytic". Theorems 4.1 and 4.2 , and the consequences of $\$ 7$ and $\$ 8$ remain valid, with the stronger conclusion that the orbits of $P_{D}$ are analytic submanifolds of $M$. How- 
ever, there is an additional fact that makes everything simpler: If $D$ is a set of analytic vector fields on $M$, then $D^{*}$ is locally of finite type. This is so because the ring $A_{m}$ of germs of analytic functions at a point $m \in M$ is noetherian, and the set of germs of vector fields at $m$ is the product $A_{m}^{n}$. It follows that $A_{m}^{n}$ is noetherian as an $A_{m}$-module. If $m \in M$, then there are finitely many elements $X^{1}$, $\ldots, X^{p}$ of $D^{*}$ such that every $X \in D^{*}$ (and, in particular, every $\left[X, X^{i}\right]$ ) can be written in some neighborhood of $m$ as $\sum_{i=1}^{p} f_{X}^{i} X^{i}$, where the functions $f_{X}^{i}$ are analytic.

In view of Theorem 8.1 , the distribution $\Delta_{D^{*}}$ has the maximal integral manifolds property. This result was proved by Nagano [7]. The method of proof based on Theorem 8.1 is due to Lobry [5].

\section{REFERENCES}

1. C. Chevalley, Theory of Lie groups. I, Princeton Math. Ser., vol. 8, Princeton Univ. Press, Princeton, N. J., 1946. MR 7, 412.

2. W. L. Chow, Über Systeme von linearen partiellen Differential-gleichungen erster Ordnung, Math. Ann. 117(1939), 98-105. MR 1, 313.

3. S. Helgason, Differential geometry and symmetric spaces, Pure and Appl. Math., vol. 12, Academic Press, New York, 1962, MR 26 \#2986.

4. R. Hermann, On the accessibility problem in control theory, Internat. Sympos. Nonlinear Differential Equations and Nonlinear Mechanics, Academic Press, New York, 1963, pp. 325-332. MR $26 \# 6891$.

5. C. Lobry, Contrôlabilité des systèmes non linéaires, SIAM J. Control 8 (1970), 573-605. MR $42 \# 6860$.

6. M. Matsuda, An integration theorem for completely integrable systems with singularities, Osaka J. Math. 5 (1968), 279-283. MR 39 \#4876.

7. T. Nagano, Linear differential systems with singularities and an application to transitive Lie algebras, J. Math. Soc. Japan 18 (1966), 398-404. MR 33 \#8005.

8. H. Sussmann and V. Jurdjevic, Controllability of nonlinear systems, J. Differential Equations 12 (1972), 95-116.

DEPARTMENT OF MATHEMATICS, UNIVERSITY OF CHICAGO, CHICAGO, ILLINOIS 60637

Current address: Department of Mathematics, Rutgers University, New Brunswick, New Jersey 08903 